\title{
Rate of Nonadherence to Antipsychotic Medications and Factors Leading to Nonadherence among Psychiatric Patients in Gondar University Hospital, Northwest Ethiopia
}

\author{
Abyot Endale Gurmu, Esileman Abdela, Bashir Allele, Ermias Cheru, and Bemnet Amogne \\ School of Pharmacy, College of Medicine and Health Sciences, University of Gondar, P.O. Box 196, Gondar, Ethiopia \\ Correspondence should be addressed to Abyot Endale Gurmu; abiymille99@yahoo.com
}

Received 14 August 2014; Revised 25 November 2014; Accepted 25 November 2014; Published 16 December 2014

Academic Editor: Xingguang Luo

Copyright (C) 2014 Abyot Endale Gurmu et al. This is an open access article distributed under the Creative Commons Attribution License, which permits unrestricted use, distribution, and reproduction in any medium, provided the original work is properly cited.

\begin{abstract}
Objective. The main aim of this study was to assess the rate of medication nonadherence among psychiatry patients at University of Gondar Hospital. Materials and Methods. Cross-sectional, descriptive method was conducted over a period of one month in May, 2013, at University of Gondar Hospital. Rate of nonadherence was computed using Medication Adherence Rating Scale questionnaire and self-reporting via a structured patient interview. Chi-square was used to determine the statistical significance of the association of variables with adherence. Result. Out of 209 respondents, 105 (50.2\%) were found to be nonadherent. Patients who were forced to take their medication against their will $(P<0.001)$, those who did not believe they require medication $(P=0.026)$, and those who discontinued their medication without consulting their prescriber $(P<0.001)$ had significant association with nonadherence. Adherence among schizophrenia was 75.7\%; psychotic was 46.7\%; bipolar disorder was $37.5 \%$; and psychosis with depression was $52.6 \%$. Reasons for nonadherence included recovery from the illness $(26.7 \%)$, seeking alternative therapy and unavailability of drugs (18.1\% each), adverse drug reaction (12.7\%), forgetfulness (10.6\%), and being busy (8.6\%). Conclusion. The observed rate of antipsychotic medication nonadherence in this study was high. Interventions to increase adherence are therefore crucial.
\end{abstract}

\section{Introduction}

Adherence to medication regimens has been monitored since the time of Hippocrates [1]. Adherence to a medication regimen is generally defined as the extent to which patients take medications as prescribed by their health care providers. It is clear that the full benefit of the many effective medications that are available will be achieved only if patients follow prescribed treatment regimens reasonably [2]. Because of the difficulties in measuring adherence, no estimate of adherence or nonadherence can be generalized, but poor adherence is to be expected in $30-50 \%$ of all patients, irrespective of disease, prognosis, or setting [3]. Study done in Washington showed that $74 \%$ of outpatients with schizophrenia stop taking neuroleptics or antipsychotics within two years of leaving a hospital and 20 to $57 \%$ patients with bipolar affective disorder are nonadherent [4].
Nonadherence to medication is known to be associated with poorer treatment outcomes, particularly in the management of chronic disease. In the treatment and management of psychotic disorders, the maximum benefit that a patient derives from these medications is highly dependent on their adherence to treatment. Although nonadherence is a ubiquitous problem in medicine, the nature of psychotic disorders makes it especially difficult for patients to adhere to treatment [1-5]. The consequences of nonadherence have been studied extensively and are significant, especially lack of disease control and hospital admissions or readmissions [6]. The impact of nonadherence on antipsychotic medication leads to diseases relapse, increased clinical and emergency room visit, and hospitalization. Nonadherent patients are over 10 times more likely to have a psychotic relapse and four times more likely to be hospitalized than adherent patients [7]. 
In Ethiopia, due to the underresourced health care system, medication nonadherence rates are potentially much higher thereby contributing to substantial worsening of disease, increased mortality, and increased health care cost [5]. Assessing medication adherence might lead to a better understanding of reasons for nonadherence in psychiatric patients and lay the groundwork for interventions aimed at increasing adherence [8]. In addition, understanding the extent of the problem is half of the solution and the result of study can help as a baseline for further study on psychiatric patient's adherence and open the door to determining various adherence and nonadherence issues.

The objective of this study was, first, to evaluate adherence rates and, second, to identify possible reasons for nonadherence to antipsychotic medications, among patients with psychiatric disorders at University of Gondar Hospital.

\section{Materials and Methods}

2.1. Study Design. A cross-sectional study design was conducted over a month period in 2013 (May 01 to May 30, 2013), on psychiatric clinic, University of Gondar Hospital (UoGH). UoGH is located $727 \mathrm{~km}$ Northwest of Addis Ababa, Ethiopia. It is one of the largest teaching institutions among federally established teaching hospitals and serving about five million populations in and around Gondar town [9].

2.2. Patient Selection Criteria. Convenience sampling was used to select patients. All patients who visited psychiatric clinic during the study period and fulfilled the inclusion criteria were recruited. Patients whose age was greater than or equal to eighteen years, who were diagnosed with psychiatric illness, who took antipsychotic medication for at least three months, who were conscious, and who volunteered to give consent were included.

2.3. Data Collection Tool. Data were collected using a structured and pretested questionnaire. The questionnaire was adapted from Medication Adherence Rating Scale (MARS) which was developed by Thompson et al., 2000 [10], for assessment of adherence in psychiatric patients and translated to local language (Amharic). It examines adherence behaviour and attitude towards medication with relatively simple scoring and includes 10 items. If the patient scored greater than or equal to six, he/she will be considered adherent and if the patient scored less than six, he/she was considered nonadherent. The questionnaire was pretested to identify potential problem of the questionnaires, unanticipated interpretations, and cultural objections to any of the questions on $11(5 \%)$ respondents having similar characteristics to the study subjects on nonparticipants. Apart from MARS questionnaire, there was also section regarding sociodemographic characteristics, treatment duration, and insight about their medication/illness. Considering those respondents who were illiterate (cannot write and read), the questionnaire was read out to them by data collectors. The diagnosis on psychiatric disorder was done as per Diagnostic and Statistical Manual of Mental disorder (http://www.dsm.org/).

2.4. Data Analysis. Data were coded, checked for completeness and consistency, and analyzed using SPSS version 20.0. Descriptive statistics were used to determine patient demographics, medication information, and adherence rates. The association between variables was calculated with chi-square test of association. Frequency tables, graphs, percentages, and means were used to display results.

2.5. Ethical Approval. The study was conducted after getting letter of permission from School of Pharmacy, University of Gondar. Confidentiality of respondents was granted by keeping the privacy while filling the questionnaire.

\section{Results}

Among two hundred and eighty patients who were approached for the study, 63 did not fulfil the inclusion criteria and 8 either did not complete interview or were not willing to be included in the study. Finally 209 were included, resulting in a response rate of $96.3 \%$. Male to female ratio was approximately $1: 1(50.2 \%$ to $49.8 \%)$ (Table 1$)$.

3.1. Clinical Characteristics of the Study Participants. Regarding the insight about their mental illness, majority 162 (77.5\%) had good understanding (59.9\% were from rural) while the rest $47(22.5 \%)$ feel they did have mental illness $(55.5 \%$ were from rural) and even did not know why they came to hospital. Of patients who reportedly had good insight into their illness, $90 \%$ believed they require medication $46.8 \%$ of patients who do not think they are ill reported that they require medication. Ninety percent of respondents have had good relationship with their mental health care provider and replied that they got sufficient information concerning their medication (Table 2).

3.2. Nonadherence Level of Respondents. Based on MARS (Medication Adherence Rating Scale), out of total respondents, half of them (50.2\%) were found nonadherent to their medication. The relative risk of residence on adherence odd ratio equals 0.962 (95\% CI; 0.766-0.206) for rural area and odd ratio equals 1.058 (95\% CI; 0.765-1.463) for urban. This implies that being from rural area had slight risk to be nonadherent even though being urban resident had no association with adherence. With regard to age, patients between 18 and 49 years old had adherence rate below 50\% whereas those above 49 years had adherence rate of $65 \%$. Correlating adherence with psychiatric disorders, schizophrenic patients were found to be the most nonadherent (75.7\%) whereas bipolar disorder accounts for the least (37.5\%) (Table 3).

Based on the association of different variables and rate of adherence, there was no statistically significant association between age, medication regimen, income, educational status, marital status, and occupational status of the respondents $(P>0.05)$. 
TABLE 1: Sociodemographic characteristics of respondents, University of Gondar Hospital, Psychiatric Clinic, May, 2013.

\begin{tabular}{lcc}
\hline Sociodemographics & Characteristics & Frequency (\%) \\
\hline \multirow{2}{*}{ Gender } & Male & $105(50.2)$ \\
& Female & $104(49.8)$ \\
\hline \multirow{3}{*}{ Age (years) } & $18-24$ & $26(12.4)$ \\
& $25-30$ & $94(45.0)$ \\
& $31-40$ & $42(20.1)$ \\
Religion & $41-50$ & $24(11.5)$ \\
& $>50$ & $23(11.0)$ \\
\hline \multirow{4}{*}{ Education level } & Orthodox & $182(87.1)$ \\
& Islam & $25(12.0)$ \\
& Protestant & $2(1.0)$ \\
\hline Residence & Illiterate & $77(33.6)$ \\
& Read and write & $11(5.3)$ \\
& Grades 1-6 & $24(11.5)$ \\
& Grades 7-10 & $46(22.0)$ \\
Garital status & Grades 11 and 12 & $14(6.7)$ \\
& Grade 12 & $37(17.7)$ \\
\hline
\end{tabular}

TABLE 2: Clinical characteristics of respondents, Gondar University Hospital, May, 2013.

\begin{tabular}{lc}
\hline Psychiatry disorder & Number (\%) \\
\hline Psychosis & $75(35.9 \%)$ \\
Major depression disorder & $60(28.7 \%)$ \\
Schizophrenia & $37(17.7 \%)$ \\
Depression with psychotic feature & $19(9.1 \%)$ \\
Bipolar disorder & $8(3.8 \%)$ \\
Others (like sleep disorder, anxiety delusional) & $10(4.8 \%)$ \\
\hline
\end{tabular}

As shown in Table 4, majority of psychiatric patients ranges in the age group of 21-30 years, of which psychosis was the Major Psychiatric disorder (39.4\%) followed by depression and schizophrenia with $23.4 \%$ each. Depression was found to be the major psychiatric illness (38.4\%) within the age group of 10-20 years, whereas psychosis was found to be the major psychiatric illness in the elderly (39.1\%).

Approximately, $90 \%$ of participants took medication from 3 months to seven years. It was found that as treatment duration increases, nonadherence rate also increases from $39.7 \%$ to $68.4 \%$ till year 7 (Figure 1).

3.3. Factors Leading to Nonadherence. Of all, 94 participants discontinued medication without consulting their prescribers for various reasons. Recovery from their illness (26\%), unavailability of drugs (18.1\%), adverse drug reactions (12.7\%), forgetfulness (10.6\%), being busy (8.6\%), and seeking

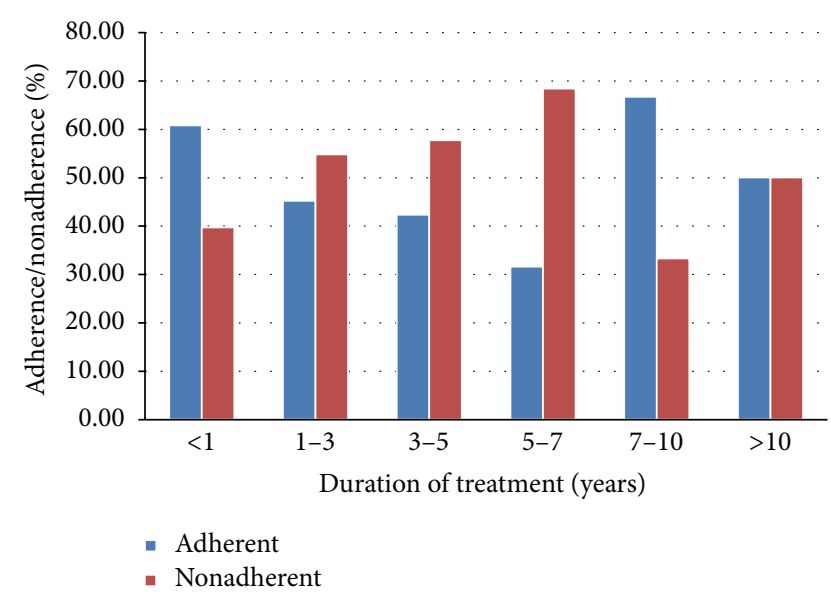

FIgURE 1: Duration on drug treatment and nonadherence rate of respondents, University of Gondar Hospital, Psychiatric Clinic, May, 2013.

alternative therapy (5.3\%) were found to be the reasons for nonadherence among respondents. With regard to those patient who had good insight into their illness, recovery from their illness (10.7\%), unavailability of drugs (10.1\%) and adverse drug reactions (6.7\%), and forgetfulness (5.6\%) were found to be the major factors for the nonadherence whereas other factors (19.1\%) and recovery from their illness (14.7\%) were the major factors for those ones who do not think they are ill (Table 5).

\section{Discussion}

This study showed that 105 (50.2\%) of respondents were nonadherent using MARS. Accessing medication adherence might lead to a better understanding level of adherence and lays groundwork for interventions and aimed at increasing adherence. Previously reported rate of adherence to antipsychotic medication in USA was ranging from 25 to $75 \%$ [11]. This was consistent with the current study.

In this study, being from rural area was found to have slight association with nonadherence rate. Another study also showed that nonadherence among rural population was more common in 1 in 5 individuals [12].

Patients who believed that they required medication were approximately 1.6 times more adherent than those who did not $(\mathrm{OR}=1.56,95 \%)$. This was consistent with a study conducted by Adams, 2000, which predicted that the association between adherence and perceived benefits of treatment is logical and may reflect the reported relationship between insight and adherence in people with mental disorders [13]. In this study, schizophrenic patients were the most nonadherent $(75.7 \%)$ compared to other psychiatry disorders while patients with bipolar disorder were the least (37.5\%). In another study rates of adherence among patients with schizophrenia were reported between 50 and 60 percent, and among those with bipolar affective disorder the rates were as low as 35 percent [1]. In a systematic review by Cramer and Rosenheck, the mean rate of medication adherence with 
TABLE 3: Nonadherence and psychotic illness among respondents, in University of Gondar Hospital, May, 2013.

\begin{tabular}{lccc}
\hline Diagnosis & Adherent (number, \%) & Nonadherent (number, \%) & Total \\
\hline Psychosis & $40(53.3)$ & $35(46.7)$ & $75(100.0 \%)$ \\
Depression & $35(58.5)$ & $25(41.7)$ & $60(100.0 \%)$ \\
Schizophrenia & $9(24.3)$ & $28(75.7)$ & $37(100.0 \%)$ \\
Psychosis with depression & $9(47.4)$ & $10(52.6)$ & $19(100.0 \%)$ \\
Bipolar disorder & $5(62.5)$ & $3(37.5)$ & $8(100 \%)$ \\
Other & $6(60)$ & $4(40)$ & $10(100.0 \%)$ \\
Total & $104(49.8)$ & $105(50.2)$ & $209(100.0 \%)$ \\
\hline
\end{tabular}

TABLE 4: Psychiatric illness in age distribution among respondents, in University of Gondar Hospital, May, 2013.

\begin{tabular}{|c|c|c|c|c|c|c|c|}
\hline \multirow{2}{*}{ Age } & \multicolumn{6}{|c|}{ Diagnosis } & \multirow{2}{*}{ Total } \\
\hline & Psychosis & Depression & Schizophrenia & Psychosis with depression & Bipolar disorder & Others & \\
\hline $10-20$ & 9 & 10 & 3 & 0 & 2 & 2 & 26 \\
\hline $21-30$ & 37 & 22 & 22 & 7 & 3 & 3 & 94 \\
\hline $31-40$ & 16 & 11 & 6 & 6 & 1 & 2 & 42 \\
\hline $41-50$ & 4 & 11 & 2 & 4 & 1 & 2 & 24 \\
\hline$>50$ & 9 & 6 & 4 & 2 & 1 & 1 & 23 \\
\hline Total & 75 & 60 & 37 & 19 & 8 & 10 & 209 \\
\hline
\end{tabular}

TABLE 5: Factors leading to nonadherence among respondents, University of Gondar Hospital, May, 2013.

\begin{tabular}{lccc}
\hline Reasons for nonadherence & Adherent & Nonadherent & Total \\
\hline Get improved & $5(18.5 \%)$ & $20(29.8 \%)$ & $5(26.6 \%)$ \\
Alternative therapy & $0(0 \%)$ & $7(10.4 \%)$ & $5(5.3 \%)$ \\
Due to ADR & $5(18.5 \%)$ & $10(14.9 \%)$ & $12.7 \%)$ \\
Due to unavailability of drugs & $7(25.9 \%)$ & $7(10.4 \%)$ & $10(10.6 \%)$ \\
Forgetfulness & $3(11.1 \%)$ & $4(5.9 \%)$ & $8(8.5 \%)$ \\
Being busy & $4(14.8 \%)$ & $14(20.8 \%)$ & $17(18 \%)$ \\
Others & $3(11.1 \%)$ & 67 & 94 \\
\hline Total & 27 & & $P .00$ \\
\hline
\end{tabular}

psychoses was 58 percent (range: 24 to 90 percent) and among those with depression the mean rate was 65 percent (range: 58 to 90 percent) [14].

In the present study, patients above 49 years old were more adherent $(65.2 \%)$ than those under 49 years old (adherence rates range from $37.5 \%$ to $51.1 \%$ ). This is consistent with expert consensus guideline and Oslin et al. studies which reported that patients who are younger were more likely to have adherence problems than older patients $[15,16]$.

Among participants, 94 (45\%) discontinued medication without mental health care provider. Given the consequences of antipsychotic discontinuation and haphazard antipsychotic use, the poor adherence rates demonstrated in this study and other studies are troubling. Fenton et al., 1997, reported that patients who discontinued antipsychotics may be two to five times more likely to relapse than other patients, leading to unnecessary suffering [17]. Robinson and colleagues reported that $82 \%$ of first-episode patients experienced at least one relapse within 5 years of followup and that patients who discontinued medication were five times more likely to relapse. It might be speculated that, after experiencing one relapse, patients would be substantially less likely to discontinue medication, so our study was particularly noteworthy in suggesting factors that might contribute to nonadherence [18].

Common barriers to adherence are under the patient's control, so attention to them is a necessary and important step in improving adherence $[19,20]$. In the current study, recovery from the illness $(26.6 \%)$ was found to be the most reason for drug discontinuation. Study done in Jimma University, Ethiopia, reported the following reasons for nonadherence among psychiatry patients: forgetfulness (36.2\%), being busy (21.0\%), and lack of sufficient information about the medication (10.0\%) [5]. In other studies typical reasons cited by patients for not taking their medications included forgetfulness (30\%), other priorities (16\%), decision to omit doses (11\%), lack of information (9\%), and emotional factors (7\%) [2]. In a study conducted by Taj et al. reasons for nonadherence included sedation (30\%), medication cost (22\%), forgetting to take medication (36\%), and inability of the physicians to explain timing and dose (92\%) or benefit of medication (76\%) [1]. 


\section{Limitation}

Being a cross-sectional design conducted at a single university hospital, our study findings cannot be generalized to all areas of the country. The self-report method used in this study to measure treatment adherence might substantially overestimate medication adherence, as it relies on patient recall.

\section{Conclusion}

This study found that nonadherence to psychiatric medication was high in the study area. Forcing patients to take their medication against their will, recovery from first episodes of their illness, and discontinuation of medication without consulting their prescribers were found to be the main contributing factors to nonadherence. Nonadherence must therefore be considered when planning treatment strategies among psychiatric patients.

\section{Conflict of Interests}

The authors declare that they have no conflict of interests.

\section{Acknowledgment}

The authors are grateful to Mr. Niguse Yigzaw, Head of Department of Psychiatry, University of Gondar, for his help during data collection.

\section{References}

[1] F. Taj, M. Tanwir, Z. Aly et al., "Factors associated with nonadherence among psychiatric patients at a tertiary care hospital, Karachi, Pakistan: a questionnaire based cross-sectional study," Journal of the Pakistan Medical Association, vol. 58, no. 8, pp. 432-436, 2008.

[2] L. Osterberg and T. Blaschke, "Adherence to medication," The New England Journal of Medicine, vol. 353, no. 5, pp. 487-497, 2005.

[3] E. Vermeire, H. Hearnshaw, P. van Royen, and J. Denekens, "Patient adherence to treatment: three decades of research. A comprehensive review," Journal of Clinical Pharmacy and Therapeutics, vol. 26, no. 5, pp. 331-342, 2001.

[4] A. Elixhauser, S. A. Eisen, J. C. Romeis, and S. M. Homan, "The effects of monitoring and feedback on compliance.," Medical care, vol. 28, no. 10, pp. 882-893, 1990.

[5] M. Alene, M. D. Wiese, M. T. Angamo, B. V. Bajorek, E. A. Yesuf, and N. T. Wabe, "Adherence to medication for the treatment of psychosis: rates and risk factors in an Ethiopian population," BMC Clinical Pharmacology, vol. 12, article 10, 2012.

[6] R. Taj and S. Khan, "A study of reasons of non-compliance to psychiatric treatment," Journal of Ayub Medical College, vol. 17, no. 2, pp. 26-28, 2005.

[7] G. Morken, J. H. Widen, and R. W. Grawe, "Non-adherence to antipsychotic medication, relapse and rehospitalisation in recent-onset schizophrenia," BMC Psychiatry, vol. 8, article 32, 2008.
[8] S. Mahaye, T. Mayime, S. S. Nkosi et al., "Medication adherence of psychiatric patients in an outpatient setting," African Journal of Pharmacy \& Pharmacology, vol. 6, pp. 608-612, 2012.

[9] WHO, "Patient safety 2013," 2013, http://www.who.int/patientsafety/implementation/apps/first_wave/ethiopia_leicester/en.

[10] K. Thompson, J. Kulkarni, and A. A. Sergejew, "Reliability and validity of a new medication adherence rating scale (MARS) for the psychoses," Schizophrenia Research, vol. 42, no. 3, pp. 241247, 2000.

[11] C. R. Dolder, J. P. Lacro, L. B. Dunn, and D. V. Jeste, "Antipsychotic medication adherence: Is there a difference between typical and atypical agents?" The American Journal of Psychiatry, vol. 159, no. 1, pp. 103-108, 2002.

[12] H. Thomas and E. Donald, "Primary medication adherence in rural population," The Journal of American Family Medicine, vol. 12, pp. 27-59, 2006.

[13] J. Adams and J. Scott, "Predicting medication adherence in severe mental disorders," Acta Psychiatrica Scandinavica, vol. 101, no. 2, pp. 119-124, 2000.

[14] J. A. Cramer and R. Rosenheck, "Compliance with medication regimens for mental and physical disorders," Psychiatric Services, vol. 49, no. 2, pp. 196-201, 1998.

[15] D. I. Velligan, P. J. Weiden, M. Sajatovic et al., "The expert consensus guideline series: adherence problems in patients with serious and persistent mental illness," The Journal of Clinical Psychiatry, vol. 70, supplement 4, pp. 1-48, 2009.

[16] D. W. Oslin, H. Pettinati, and J. R. Volpicelli, "Alcoholism treatment adherence: older age predicts better adherence and drinking outcomes," American Journal of Geriatric Psychiatry, vol. 10, no. 6, pp. 740-747, 2002.

[17] W. S. Fenton, C. R. Blyler, and R. K. Heinssen, "Determinants of medication compliance in schizophrenia: empirical and clinical findings," Schizophrenia Bulletin, vol. 23, no. 4, pp. 637-651, 1997.

[18] D. Robinson, M. G. Woerner, J. M. J. Alvir et al., "Predictors of relapse following response from a first episode of schizophrenia or schizoaffective disorder," Archives of General Psychiatry, vol. 56, no. 3, pp. 241-247, 1999.

[19] S. Magura, A. Roseblum, and C. Fong, "Factor associated with medication adherence among psychiatric outpatients at substance abuse risk," The Open Addiction Journal, vol. 4, pp. 58-64, 2011.

[20] K. S. Latha, "The noncompliant patient in psychiatry: the case for and against covert/surreptitious medication," Mens Sana Monographs, vol. 8, no. 1, pp. 96-121, 2010. 


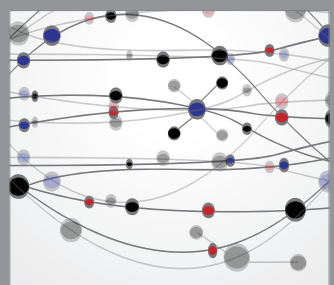

The Scientific World Journal
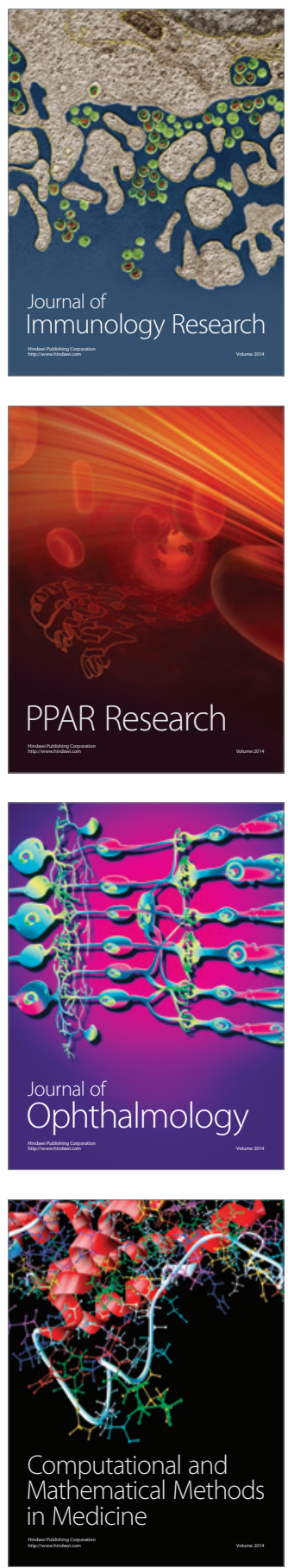

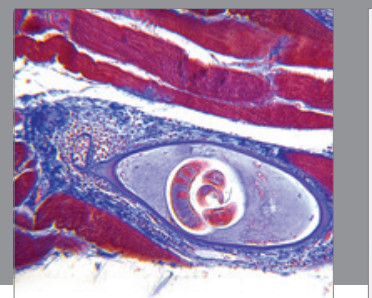

Gastroenterology

Research and Practice
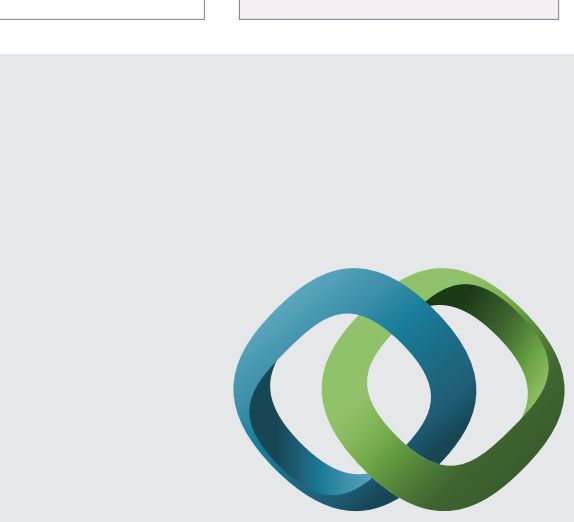

\section{Hindawi}

Submit your manuscripts at

http://www.hindawi.com
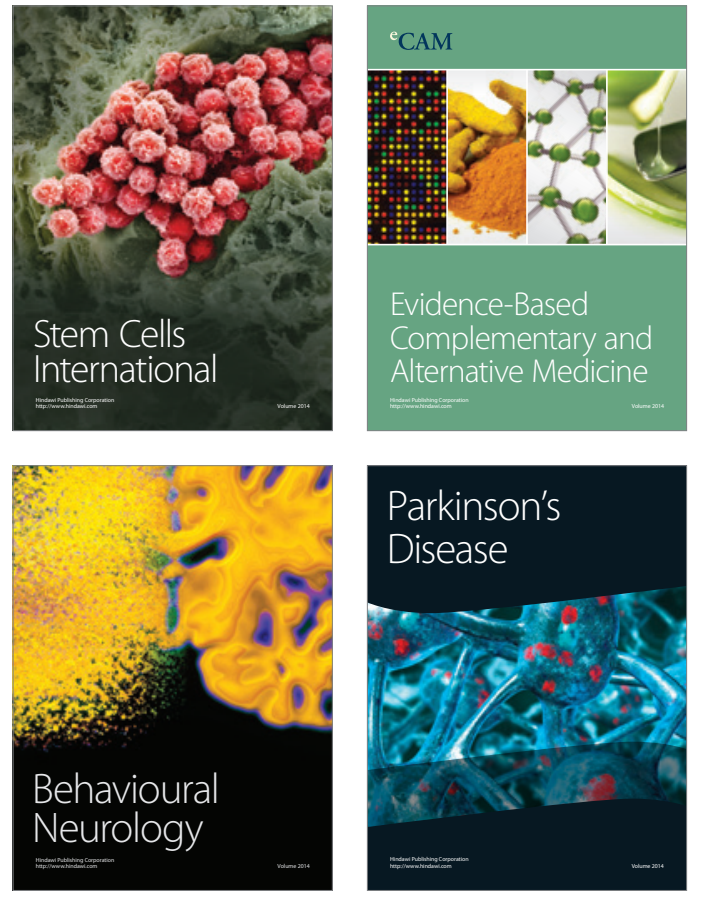
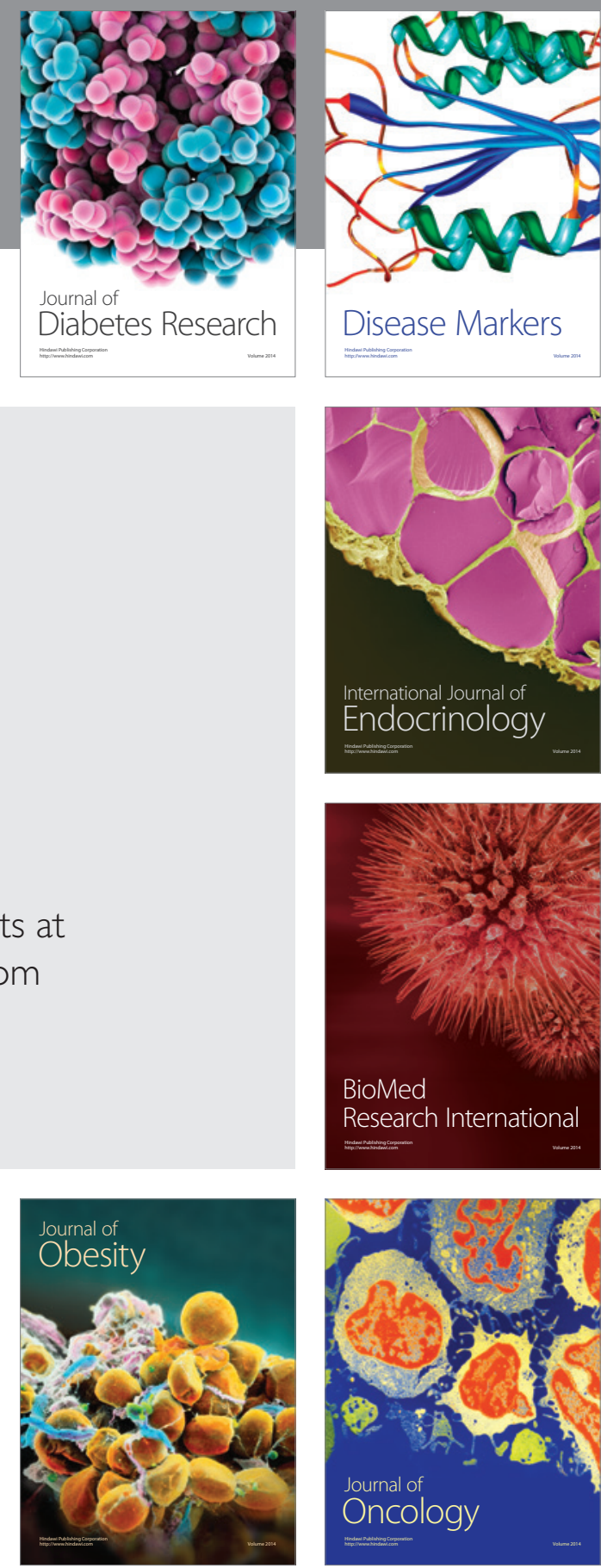

Disease Markers
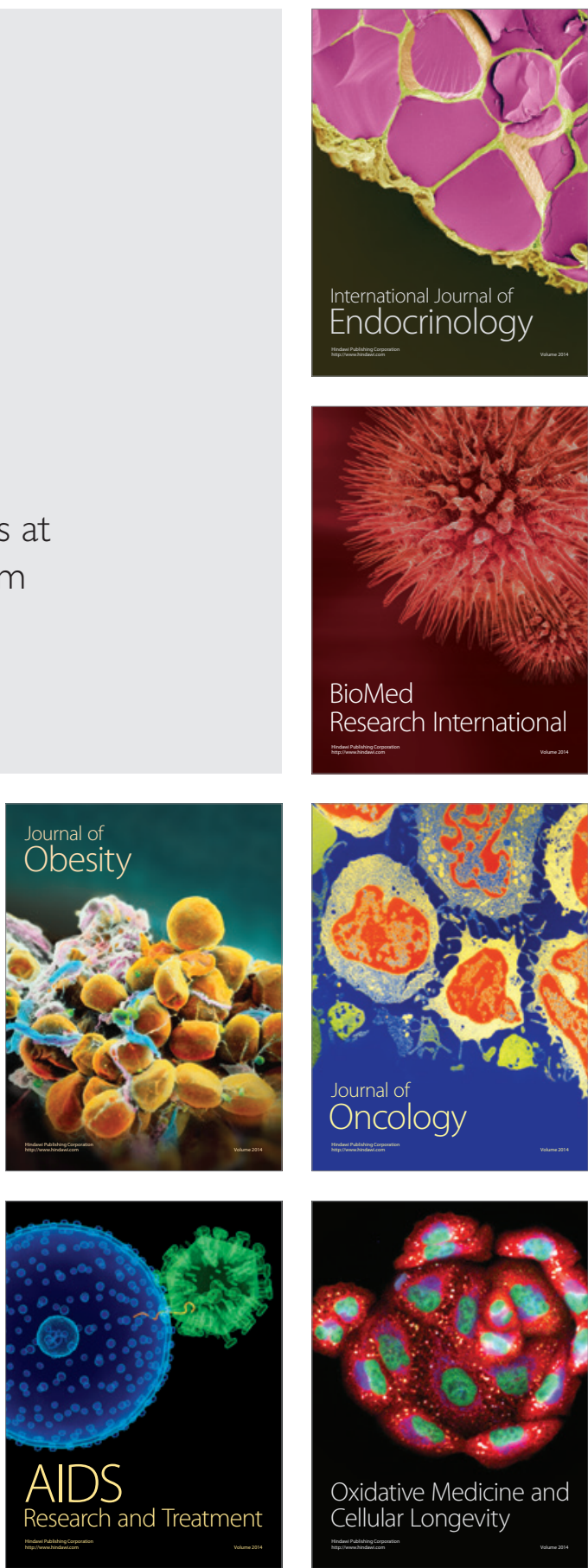